\title{
Research of YBCO Superconductor Coating on Austenitic Stainless Steel by Electrophoretic Deposition
}

\author{
S. Sato, S. Koura \\ Department of Applied Chemistry, Chiba Institute of Technology \\ Narashino, Chiba 275-0016, JAPAN
}

\begin{abstract}
Yttrium-based superconductors (YBCO) haven't been widely applied. One reason is because of its high cost. In this research, we replaced the substrate and the fabrication method with a lower and found the possibility of fabricating high-performance superconducting wires. Specifically, the YBCO layer was fabricated by electrophoretic deposition (EPD). However, this method requires calcination. It has been known that $\mathrm{Fe}$ in the substrate diffuses into the YBCO layer during calcination, and this phenomenon causes deterioration of $\mathrm{YBCO}$ performance. Therefore, $\mathrm{CeO} 2$ was fabricated as a diffusion prevention layer between the substrate and the YBCO layer. It showed the possibility of preventing the diffusion of $\mathrm{Fe}$. Mean while, a textured $\mathrm{CeO} 2$ layer was fabricated to enhance the properties of the superconductor. It was also shown that controlling the current may provide preferential orientation.
\end{abstract}

\section{Introduction}

Yttrium-based superconductors (YBCO) are expected to be applied to transmission lines (1). But it have not been widely used, one of the reason is that the substrate and the film fabrication cost are too expensive. In order to solve these problems, using stainless steel (SUS) and an electrophoretic deposition (EPD) were adopted as a new system.

Currently, Hastelloy is used for the substrate of superconducting wire (2). Since this alloy is expensive, we focus on SUS which is reasonable than Hastelloy and has various types with different compositions. So it is possible to use the appropriate adopting the environment for low cost. For these reasons, we have selected stainless steel as the alternative to Hastelloy. However, it has been reported that stainless steel has low oxidation resistance in high-temperature (3). It may cause to decrease the conduction performance as a wire (4). We would try to solve this problem on this study. On the other hand, fabricated films by EPD is generally known to have low adhesion (5). Therefore, we also aimed to improve the adhesion between substrate and films. From the above we focus on two points and create the new superconducting wires.

In addition, physical properties of superconductors is known to improve the characteristics by controlling the crystallite plane orientation, plating methods that preferential oriented layers can be obtained by controlling the current density has been reported (6). From here, we also studied to fabricating textured $\mathrm{CeO}_{2}$ as intermediate layer by controlling the current density on EPD and to further improve the wire properties. 


\section{Experimental}

\subsection{Fabricating the YBCO layer.}

Fig.1 shows the overview on this experiment until fabricating oxide film. The YBCO calcined powder is synthesized by solid-state chemical reaction (7). The molar ratio of $\mathrm{Y}$, $\mathrm{Ba}$, and $\mathrm{Cu}$ was prepared at $1: 2: 3$ using $\mathrm{Y}_{2} \mathrm{O}_{3}, \mathrm{BaCO}_{3}$ and $\mathrm{CuO}$. Then it was heated up to $880{ }^{\circ} \mathrm{C}$ for 10 hours following Fig.2(a) and crushed. This process was performed three times in total. The powder was analyzed by X-ray diffraction (XRD, Rigaku RINT-2100).

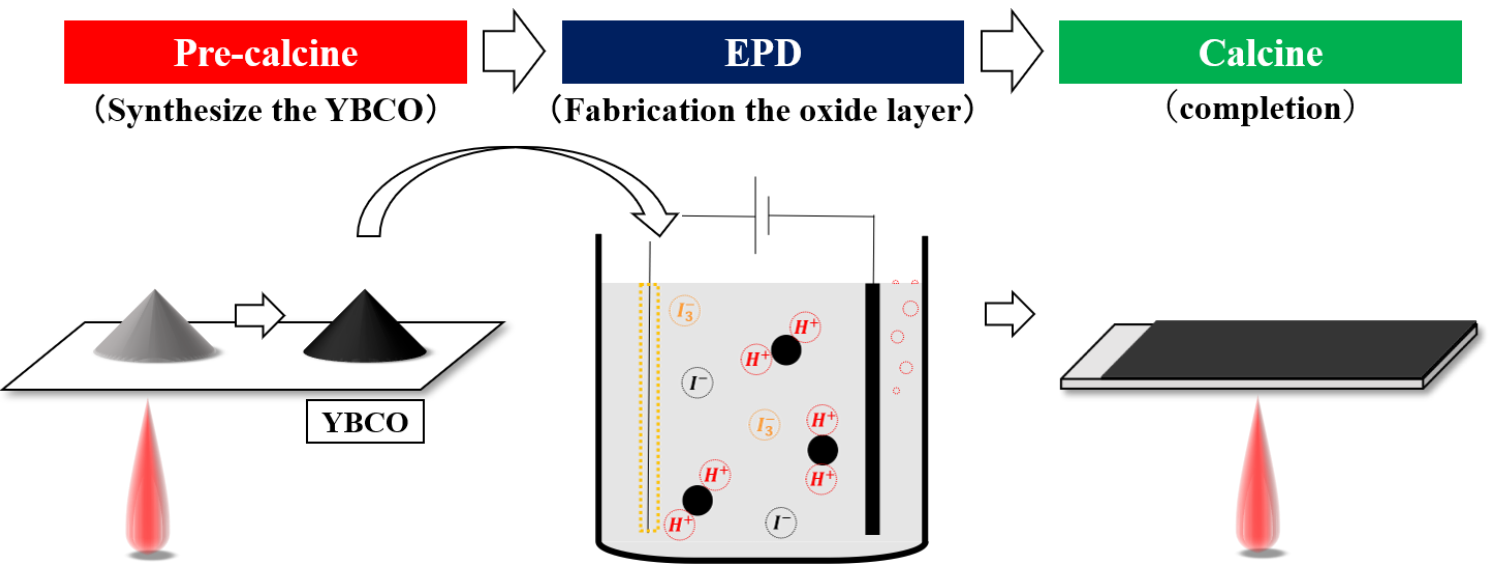

Fig.1 Overview on this experiment.

EPD was used to fabricating $\mathrm{YBCO}$ or $\mathrm{CeO}_{2}$ film. The EPD bath consists of a SUS both electrode and oxide suspension. Acetone were employed as a media and the concentration of $\mathrm{YBCO}$ or $\mathrm{CeO}_{2}$ was $10 \mathrm{~g} / 1,0.1 \sim 0.2 \mathrm{~g} / \mathrm{l}$ of $\mathrm{I}_{2}, 1 \mathrm{ml} / 1$ of water with a magnetic stirrer at a rotation speed of $500 \mathrm{rpm}(8)$. After $10 \mathrm{~min}$ of ultrasonication, the suspensions were ready to use (9). The distance between the both electrodes was about $2 \mathrm{~cm}$ and constant voltage mode $600 \mathrm{~V}$ was applied with a REGULATED DC POWER SUPPLY (TSS Takasago, TP0650-01D) for 60 seconds, and coatings the oxide layer on the cathode.

Multilayer coatings were heated up to $950{ }^{\circ} \mathrm{C}$ for 6 hours following Fig.2(b). After that, it was analyzed by electron probe microanalysis (EPMA, Shimadzu EPMA-1720) to know the film thickness and diffusion of elements for the interface between substrate and oxide films.
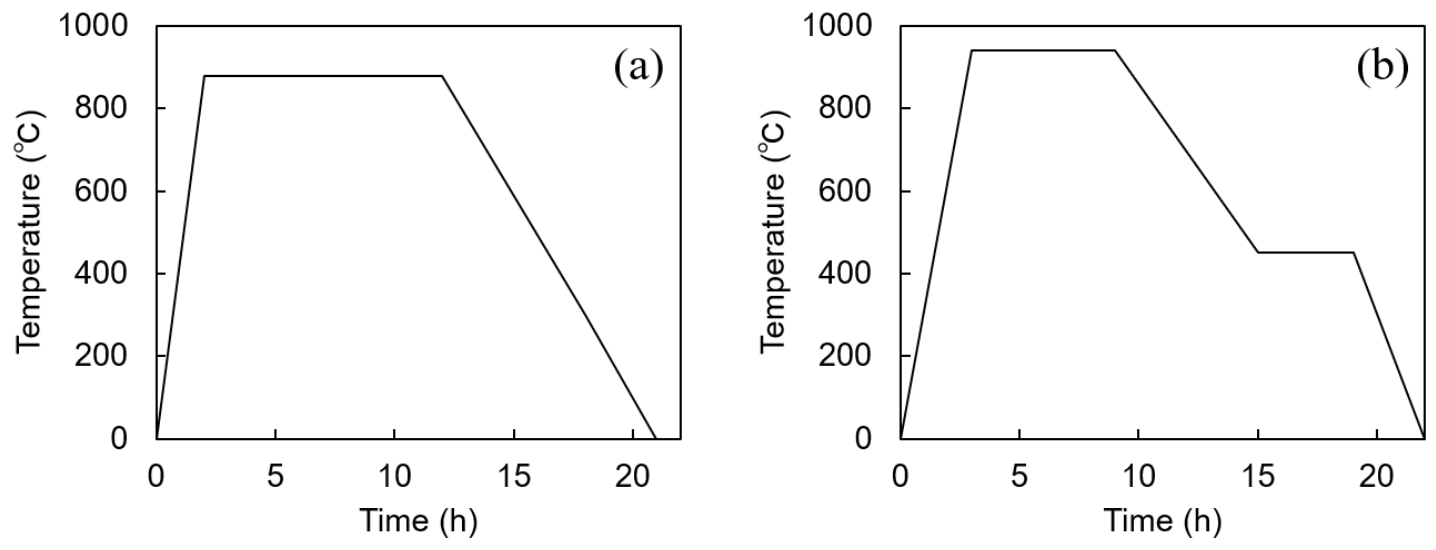

Fig.2 (a)Heat pattern of pre-calcine and (b)heat pattern of calcine. 


\subsection{Fabricating oriented $\mathrm{CeO}_{2}$ layer.}

The EPD bath consists is almost the same as above. But constant current mode was changed to constant voltage mode and current density was controlled $0.5 \sim 5.0 \mathrm{~mA} / \mathrm{cm}^{2}$ and textured $\mathrm{CeO}_{2}$ layer was synthesized. Orientation was analyzed by X-ray diffraction (XRD, Rigaku SmartLab).

\section{Results and Discussion}

\subsection{Identification of YBCO calcine powder}

Fig.3 shows the XRD results for pure YBCO (Fig.3(a)) and calcined powder (Fig.3(b)) was analyzed by XRD (10). The diffraction peak of $\phi_{103}, \phi_{020}$ and $\phi_{123}$ were confirmed. From this, Obtained peaks almost match with the standard YBCO and the powder was identified as YBCO. But $\phi_{010}$ and $\phi_{113}$ of the calcined powder were weaker than pure YBCO. It can be considered that $\mathrm{O}$ contained in powder is less than the pure YBCO and this problem causes a decrease in the characteristics of superconducting wire. It may be solved by calcinating in oxygen atmosphere.

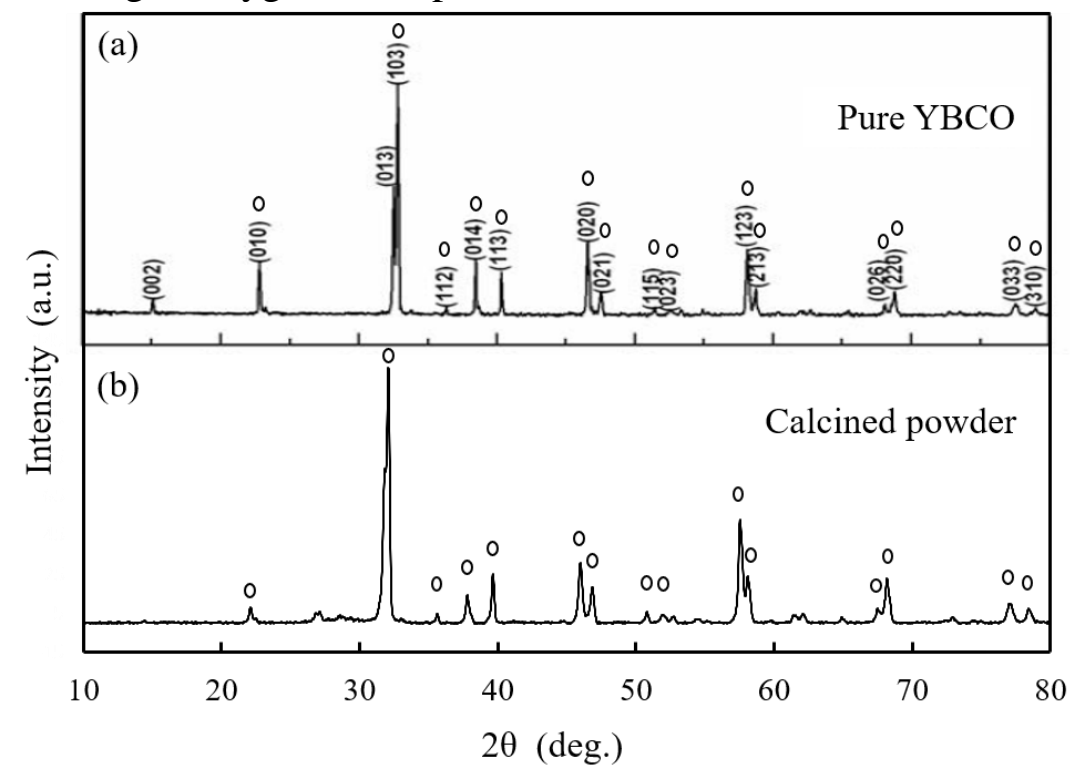

Fig.3 (a)X-ray diffraction patterns of pure YBCO and (b) calcined powder.

\subsection{Examination of optimal EPD conditions}

At the first EPD, Peeling off was confirmed on YBCO film (Fig.4(a)). This reason was considered that hydrogen generated from the cathode. The reaction formula was shown below (11).

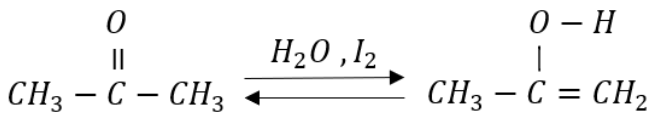

$$
\begin{aligned}
& \begin{array}{ccc}
\mathrm{O} & -\mathrm{H} \\
\mathrm{I} & \stackrel{O}{\mathrm{I}} \\
\mathrm{CH}_{3}-\mathrm{C}=\mathrm{CH}_{2} \stackrel{\mathrm{I}_{2}}{\longrightarrow} \mathrm{CH}_{3}-\mathrm{I} \\
\mathrm{C}
\end{array}
\end{aligned}
$$


The hydrogen is derived from hydrogen ions and it was generated by the catalytic action of enol type acetone and iodine present in the solution. Therefore, in order to suppress the hydrogens, iodine or enol type acetone concentration was reduced than before. In this study,
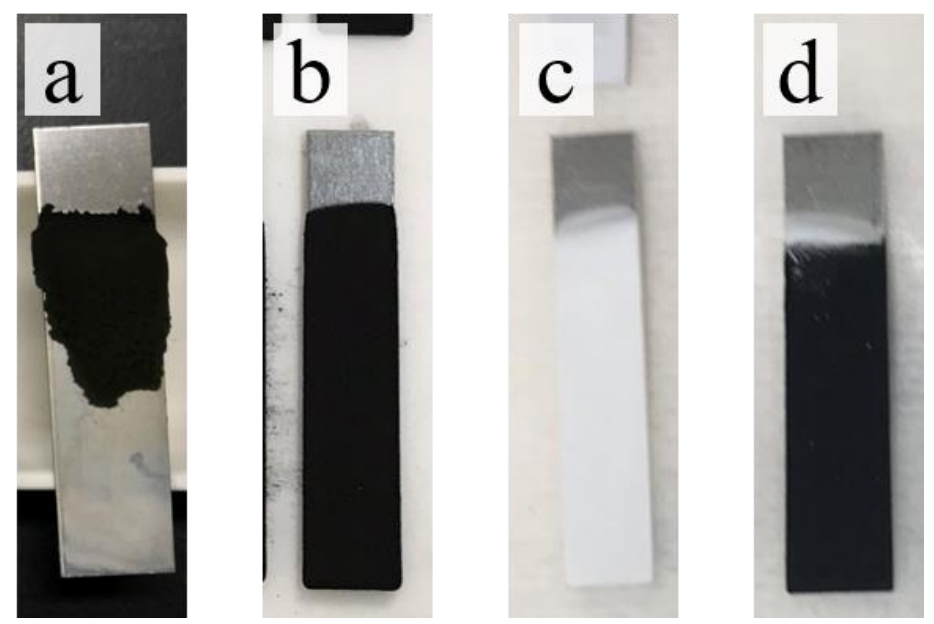

Fig.4 (a) Samples fabricated by changing EPD conditions. $\mathrm{I}_{2} 0.2 \mathrm{~g} / \mathrm{l}$ conditions, (b) $\mathrm{I}_{2} 0.1$ $\mathrm{g} / \mathrm{l}$ conditions, (c) fabricated $\mathrm{CeO}_{2}$ film, (d) deposited of $\mathrm{CeO}_{2}$ and $\mathrm{YBCO}$.

Iodine concentration was reduced from $0.2 \mathrm{~g} / 1$ to $0.1 \mathrm{~g} / \mathrm{l}$. As a result of above conditions, the film didn't peel off (Fig.4(b)). From this, the reason of film peeling was generated hydrogen from the cathode, and $0.1 \mathrm{~g} / 1$ iodine concentration is suitable for EPD when fabricating the $\mathrm{YBCO}$ film. $\mathrm{CeO}_{2}$ film was also fabricating in the same conditions(Fig.4(c)). Finally, $\mathrm{CeO}_{2}$ and $\mathrm{YBCO}$ were also deposited on SUS (Fig.4(d)) and fabricating of multilayer on substrate.

Fig.5 shows the electron micrograph for YBCO deposited on SUS (Fig.5 (a)) and $\mathrm{CeO}_{2}$, YBCO deposited on SUS (Fig.5(b)). Comparing each layers in Fig.5(b), although the $\mathrm{CeO}_{2}$ layer is densely deposited, but YBCO layer is not densely and many voids can be confirmed. It is considered that the particle size of YBCO is too large. Therefore, the size will be reduced in next time.
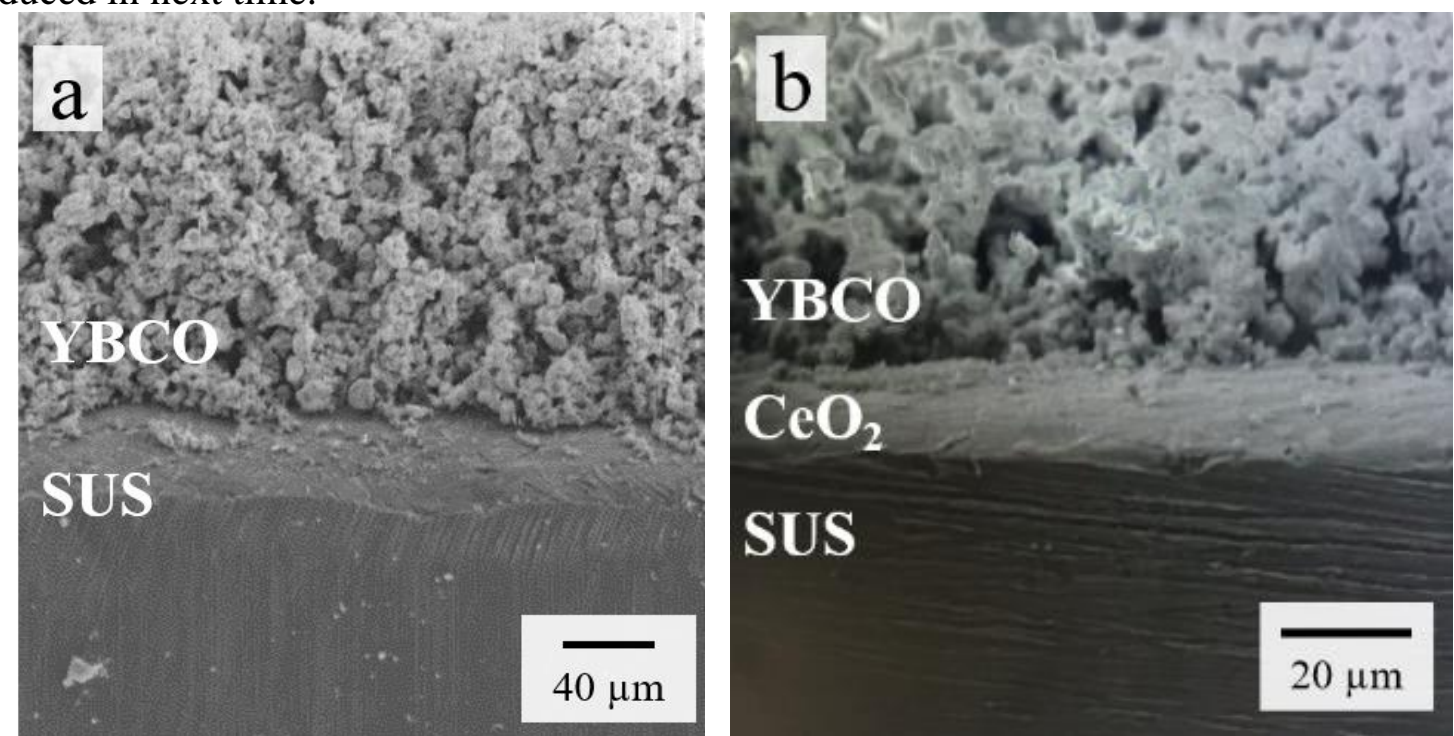

Fig.5 (a) SEM images of YBCO deposited SUS and (b) fabricating $\mathrm{CeO}_{2}$ layer between SUS and YBCO. 


\subsection{Analyzation of the elemental for main calcination}

Fig.6 shows analyzed elemental mapping with EPMA between the SUS and the YBCO layers after heating up to $950{ }^{\circ} \mathrm{C}$. At this time, the existence of $\mathrm{Fe}$ was confirmed in the YBCO layer . YBCO is consisted of $\mathrm{Y}, \mathrm{Ba}, \mathrm{Cu}$, and $\mathrm{O}$. Fe isn't consisted in YBCO. Including $\mathrm{Fe}$ is only SUS in the sample. From above, it can be considered that Fe was diffused from the SUS layer to the YBCO layer by main calcination. While $\mathrm{O}$ was also detected in the SUS layer. However it isn't included O. Therefore, it is considered that the diffusion of $\mathrm{O}$ occurred from the YBCO layer to the SUS layer, contrary to the diffusion of Fe. From this results, intermediate layer between the SUS and the YBCO layer is necessary to prevent the diffusion of $\mathrm{Fe}$ and $\mathrm{O}$.

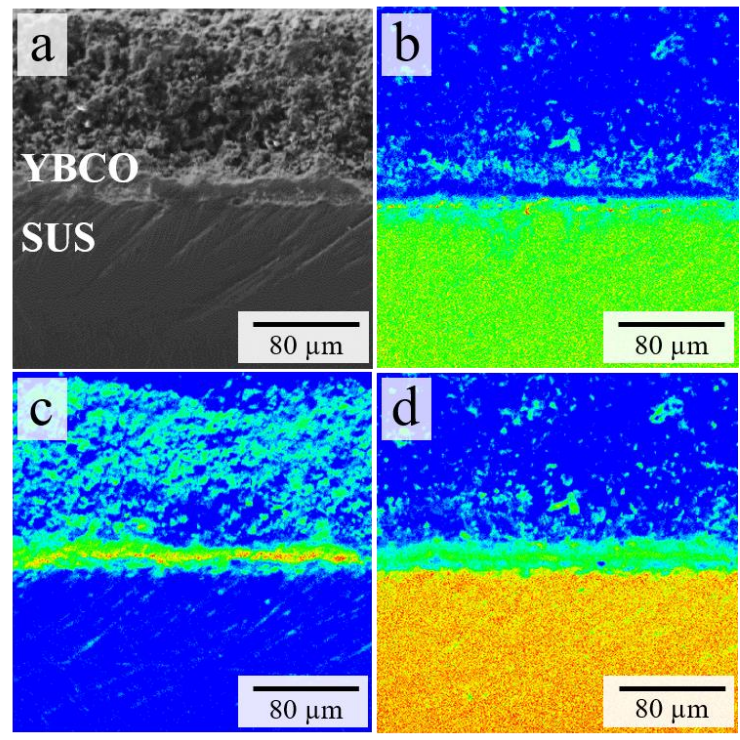

Fig.6 (a)Electron probe microanalysis images of sample deposited YBCO on SUS, (b) elemental mapping of $\mathrm{Fe}$, (c) $\mathrm{O}$, (d) $\mathrm{Cr}$

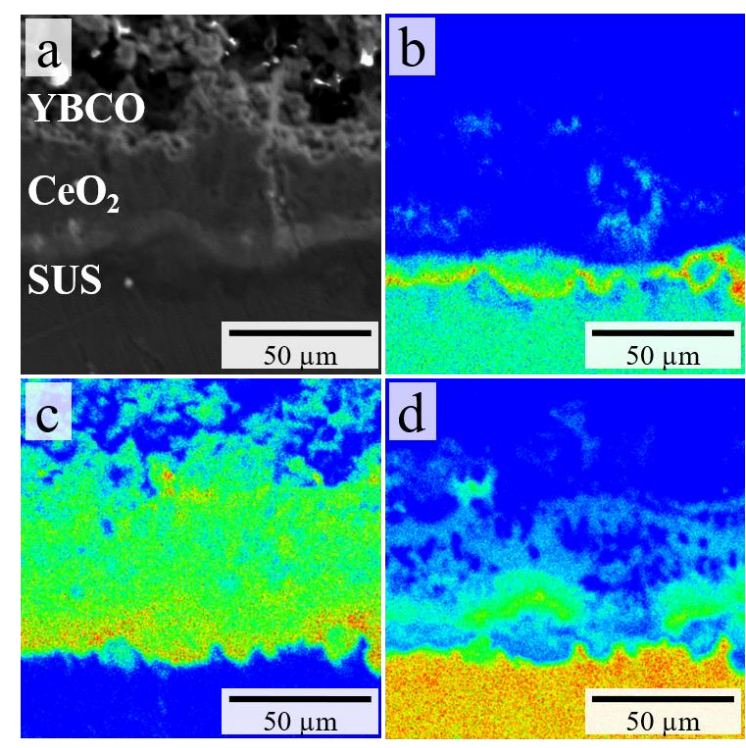

Fig.7 (a) Electron probe microanalysis images of sample deposited YBCO and $\mathrm{CeO}_{2}$ on SUS, (b) elemental mapping of $\mathrm{Fe}$, (c) $\mathrm{O}$, (d) $\mathrm{Cr}$

\subsection{Preventing the elemental for main calcination}

Elemental diffusion was caused at deposited YBCO on SUS. To prevent this phenomenon, $\mathrm{CeO}_{2}$ was fabricated as an intermediate layer between SUS and YBCO. Fig.7 shows analyzed elemental mapping with EPMA between the SUS, $\mathrm{CeO}_{2}$ and the $\mathrm{YBCO}$ layer. When comparing Fig. 6 and 7, it can be confirmed that the element diffusion of Fe from SUS to YBCO layer did not occur in Fig.7. From this, Elemental diffusion of $\mathrm{Fe}$ which reduces the performance of superconductor was not occurred by $\mathrm{CeO}_{2}$ layer, and the possibility that a high-performance superconducting wire can be produced by EPD was suggested.

\subsection{Fabricating oriented $\mathrm{CeO}_{2}$ layer control by current control}

Fig. 8 shows the XRD results for SUS, $\mathrm{CeO}_{2}$ deposited on SUS under $0.5 \mathrm{~mA} / \mathrm{cm}^{2}$ and 5.0 $\mathrm{mA} / \mathrm{cm}^{2}$. The diffraction peak of the planes $\phi_{006}=20.9^{\circ}, \phi_{111}=43.5^{\circ}, \phi_{200}=50.7^{\circ}$ and $\phi_{220}=74.5^{\circ}$ are derived from Fe in SUS. Peak of $\phi 006$ is confirmed in all of patterns. But peaks of $\phi_{111}, \phi_{200}$ and $\phi_{220}$ weren't confirmed in $0.5 \mathrm{~mA} / \mathrm{cm}^{2}$. As a reason for this, it can be considered that the thickness of the film was affected. because $\mathrm{CeO}_{2}$ layer of 0.5 
$\mathrm{mA} / \mathrm{cm}^{2}$ was thicker than $5.0 \mathrm{~mA} / \mathrm{cm}^{2}$ and X-rays did not reach the substrate, these peaks weren't confirmed.

On the other hand, the crystallite orientation, towards $5.0 \mathrm{~mA} / \mathrm{cm}^{2}$ compared to $0.5 \mathrm{~mA} / \mathrm{cm}^{2}$ tends to peak $\phi_{220}$ and $\phi_{311}$ is increased. From this, it was suggested that the crystallite orientation could be controlled by controlling the current.

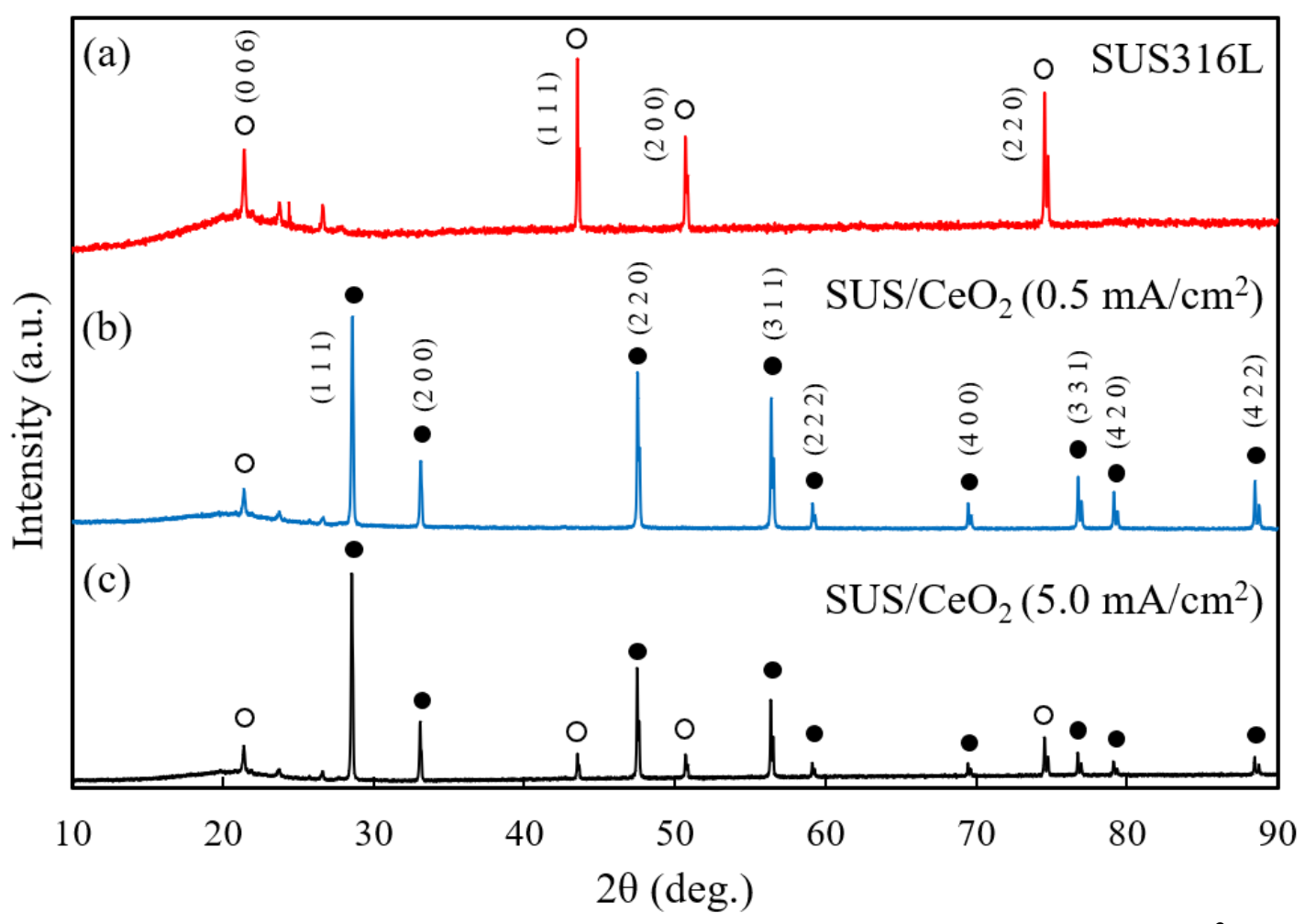

Fig.8 (a) X-ray diffraction patterns of SUS (b) deposited $\mathrm{CeO}_{2}$ on $\mathrm{SUS}\left(0.5 \mathrm{~mA} / \mathrm{cm}^{2}\right)$, (c) deposited $\mathrm{CeO}_{2}$ on SUS $\left(5.0 \mathrm{~mA} / \mathrm{cm}^{2}\right)$

\section{Conclusion}

The substrate material was replaced from Hastelloy to SUS, and by further fabricating EPD, considering the production of inexpensive and high-performance superconductor wire than conventional, the following findings were obtained.

1. The peak of $\phi_{010}$ and $\phi_{113}$ are weak, but YBCO of the same composition as powder was obtained roughly.

2. Concentration conditions of optimal Iodine was found to be $0.1 \mathrm{~g} / 1$ when depositing of YBCO by EPD.

3. YBCO was deposited on SUS by EPD. But it was confirmed that the elemental diffusion of Fe and $\mathrm{O}$ occurs. On the other hand, it was clarified that it is possible to prevent these by applying $\mathrm{CeO}_{2}$ to the middle layer.

4. It was suggested that it was possible to control the crystallite orientation of oxides deposited by controlling the current in EPD.

From the above, the possibility that high-performance superconducting wire using EPD can be produced was obtained 


\section{References}

1. Y. Shiohara, Fujikura Technical Review, 129, 1 (2016)

2. M. Kisu, T. Iwakuma and Y. Shiohara et al, OYO-BUTURI, 71, 30 (2002)

3. H. Kowaka, Journal of The Society of Materials Science. Japan, 23, 36 (1974)

4. Y. Yamada, T. Hattori, Phisica C, 335, 80 (2000)

5. Y. Takayama, N. Koura and Y. Idemoto et al, Journal of Ceramic Society of Japan, 107, 437 (1999)

6. Y. Shinkai, T.Nagaishi and M. Konishi, SEI Technical Review, 174, 105 (2009)

7. M. Ono, M. Koshimura and M. Abe, Journal of Magnetics Society of Japan, 12, 431 (1988)

8. N. Minami and N. Koura, Surface Finishing Society of Japan, 43, 93 (1992)

9. T. Uchikoshi, T. S. Suzuki and H. Okuyama et al, Journal of the European Ceramic Society, 24 (2004)

10. A. K. Jha, N. Khare, Physica C, 469, 811 (2009)

11. N. Koura and H. Shouji, Surface Finishing Society of Japan, 42, 48 (1991) 